\title{
Characterizing the radial oxygen abundance distribution in disk galaxies ${ }^{\star}$
}

\author{
I. A. Zinchenko ${ }^{1,2}$, A. Just ${ }^{2}$, L. S. Pilyugin ${ }^{1,2}$, and M. A. Lara-Lopez ${ }^{3}$ \\ 1 Main Astronomical Observatory, National Academy of Sciences of Ukraine, 27 Akademika Zabolotnoho St., 03680 Kyiv, Ukraine \\ e-mail: zinchenko@mao.kiev.ua \\ 2 Astronomisches Rechen-Institut, Zentrum für Astronomie der Universität Heidelberg, Mönchhofstr. 12-14, \\ 69120 Heidelberg, Germany \\ 3 Dark Cosmology Centre, Niels Bohr Institute, University of Copenhagen, Juliane Maries Vej 30, 2100 Copenhagen, Denmark
}

Received 2 October 2018 / Accepted 13 January 2019

\begin{abstract}
Context. The relation between the radial oxygen abundance distribution (gradient) and other parameters of a galaxy such as mass, Hubble type, and a bar strength, remains unclear although a large amount of observational data have been obtained in the past years.

Aims. We examine the possible dependence of the radial oxygen abundance distribution on non-axisymmetrical structures (bar/spirals) and other macroscopic parameters such as the mass, the optical radius $R_{25}$, the color $g-r$, and the surface brightness of the galaxy. A sample of disk galaxies from the third data release of the Calar Alto Legacy Integral Field Area Survey (CALIFA DR3) is considered. Methods. We adopted the Fourier amplitude $A_{2}$ of the surface brightness as a quantitative characteristic of the strength of nonaxisymmetric structures in a galactic disk, in addition to the commonly used morphologic division for $\mathrm{A}$, AB, and $\mathrm{B}$ types based on the Hubble classification. To distinguish changes in local oxygen abundance caused by the non-axisymmetrical structures, the multiparametric mass-metallicity relation was constructed as a function of parameters such as the bar/spiral pattern strength, the disk size, color index $g-r$ in the Sloan Digital Sky Survey (SDSS) bands, and central surface brightness of the disk. The gas-phase oxygen abundance gradient is determined by using the $\mathrm{R}$ calibration.

Results. We find that there is no significant impact of the non-axisymmetric structures such as a bar and/or spiral patterns on the local oxygen abundance and radial oxygen abundance gradient of disk galaxies. Galaxies with higher mass, however, exhibit flatter oxygen abundance gradients in units of dex/kpc, but this effect is significantly less prominent for the oxygen abundance gradients in units of dex $/ R_{25}$ and almost disappears when the inner parts are avoided $\left(R>0.25 R_{25}\right)$. We show that the oxygen abundance in the central part of the galaxy depends neither on the optical radius $R_{25}$ nor on the color $g-r$ or the surface brightness of the galaxy. Instead, outside the central part of the galaxy, the oxygen abundance increases with $g-r$ value and central surface brightness of the disk.
\end{abstract}

Key words. galaxies: abundances - ISM: abundances - $\mathrm{H}$ II regions

\section{Introduction}

It is known that the chemical abundances of heavy elements decrease with increasing galactocentric distance in the disks of our Galaxy and other nearby spiral galaxies (e.g., Mayor 1976; Searle 1971; Peimbert 1979; Shields \& Searle 1978; Belfiore et al. 2017). This trend is usually referred to as the radial metallicity gradient (RMG). The oxygen abundance gradient in nearby galaxies, traced by H II regions, ranges from $\sim 0 \mathrm{dex} \mathrm{kpc}^{-1}$ to -0.1 dex kpc $^{-1}$ (e.g., Vila-Costas \& Edmunds 1992; Zaritsky et al. 1994; Sánchez et al. 2014; Pilyugin et al. 2014a). However, the origin of the gradient and its relation to other macroscopic parameters of galaxies are still open for discussion.

During the past decade, significant progress in the understanding of the main tendencies of chemical abundance distribution in galaxies became possible through integral field spectroscopy (IFS) data that were obtained in frameworks of large galaxy surveys. One such survey is the Calar Alto Legacy Integral Field Area Survey (CALIFA; Sánchez et al. 2012). Using oxygen abundance measurements in a large number of $\mathrm{H}$ II

\footnotetext{
$\star$ Table 1 is also available at the CDS via anonymous ftp to cdsarc.u-strasbg.fr (130.79.128.5) or via http://cdsarc. u-strasbg.fr/viz-bin/qcat?]/A+A/623/A7
}

regions in more than 300 spiral galaxies from the CALIFA survey, Sánchez et al. (2014) showed that disk galaxies in the local Universe exhibit a common characteristic gradient in the oxygen abundance up to two effective disk radii, regardless of the galaxy parameters. The distribution of the abundance gradients is Gaussian around some mean value. Therefore, Sánchez et al. (2014) suggested that the scatter around the mean value could be a result of random fluctuations.

Sánchez et al. (2014) claimed that this result contradicts several previous observational studies that concluded that the slope of the gas-phase abundance gradient is related to other galactic properties, such as

- morphology: the slopes of early-type spirals are more shallow than those of late-type galaxies (e.g., McCall et al. 1985; Vila-Costas \& Edmunds 1992);

- mass: the slopes of more massive spirals are more shallow than those of less massive galaxies (e.g., Zaritsky et al. 1994; Martin \& Roy 1994; Garnett 1998);

- presence of a bar: the slopes of barred galaxies are more shallow than than those of unbarred galaxies (e.g., Vila-Costas \& Edmunds 1992; Zaritsky et al. 1994; Roy 1996);

- interaction stage of the galaxies: evolved mergers present shallower slopes (e.g., Rich et al. 2012). 
In contrast, many authors found no correlation between abundance gradient (expressed in $\mathrm{dex} / R_{25}$ ) and morphology (Zaritsky et al. 1994; Pilyugin et al. 2014a; Sánchez et al. 2014; Sánchez-Menguiano et al. 2016) and stellar mass (Ho et al. 2015; Sánchez-Menguiano et al. 2016, 2018). However, for the metallicity gradients expressed in dex/kpc, Ho et al. (2015) found that galaxies with lower mass tend to have steeper metallicity gradients on average.

Using the CALIFA survey, Sánchez-Blázquez et al. (2014) found no difference in the slope of the stellar-phase metallicity gradient for barred and unbarred galaxies. The same conclusion was made by Cheung et al. (2015), who analyzed stacked spectra from the Sloan Digital Sky Survey (SDSS).

Based on a sample of 550 nearby galaxies with integral field unit (IFU) data from the SDSS IV Mapping Nearby Galaxies at Apache Point Observatory survey (SDSS/MaNGA; Bundy et al. 2015; Blanton et al. 2017), it has recently been found that the radial metallicity gradient steepens when the stellar mass increases up to $\log \left(M / M_{\odot}\right)=10.5$, but flattens for galaxies with higher masses (Belfiore et al. 2017). However, using the same data from the SDSS-IV MaNGA survey, Lian et al. (2018) found that the stellar metallicity gradients tend to be mass dependent with steeper gradients in more massive galaxies, but they found no clear mass dependence for the gas metallicity gradient. Moreover, a recent analysis of IFU data suggests that galaxies can present a different behavior of the radial abundance gradient in the inner and outer parts (Sánchez et al. 2014; SánchezMenguiano et al. 2016, 2018; Belfiore et al. 2017).

According to the current observational picture presented above, despite the wide range of metallicity gradients exhibited by galaxies, only a few studies point out the connection between radial metallicity gradient and galaxy mass. Thus, there is no solid confirmation of the correlation between oxygen abundance gradient and galaxy parameters (with the exception of mergers).

On the other hand, theoretical simulations based on pure N-body galactic disk dynamics and self-consistent chemodynamical models produce an appreciable influence of nonaxisymmetric structures (bar and spiral patterns) in the galactic disk, and interactions between galaxies. Several theoretical studies have suggested various mechanisms of gas and star mixing in barred galaxies (Athanassoula 1992; Sellwood \& Binney 2002; Schönrich \& Binney 2009; Minchev \& Famaey 2010; Di Matteo et al. 2013; Minchev et al. 2013, 2014) that might lead to the flattening of the oxygen abundance distribution. This scenario is consistent with observational results (Ruiz-Lara et al 2017) based on the analysis of the stellar content in a sample of CALIFA galaxies. Thus, the redistribution of heavy elements produced by the radial migration of stars and/or interstellar gas along the galactic disk is assumed to be the cause of a flattening of the metallicity gradient for the intermediate-age stellar populations and interstellar gas. However, Grand \& Kawata (2016) demonstrated that corotating spiral arms do not change the radial metallicity gradient.

Zinchenko et al. (2016) analyzed the azimuthal variation of the oxygen abundance in CALIFA galaxies and found no significant asymmetry in the azimuthal abundance distribution. In this paper, we investigate the possible dependence of the oxygen abundance gradient in disk galaxies on a bar/spiral and other parameters of the galaxy, such as mass, size, color index, and surface brightness. We also study the mass-metallicity relation for the local oxygen abundance in the center and in intermediate and outer parts of the galaxy as a multivariable relation including size, color index, surface brightness, and the bar/spiral pattern strength.

\section{Data}

\subsection{Sample}

We used publicly available spectra from the integral field spectroscopic CALIFA survey data release 3 (DR3; Sánchez et al. 2016, 2012; Walcher et al. 2014) based on observations with the Potsdam Multi-Aperture Spectrophotometer (PMAS) and a specialized fiber-bundle, called PPAK (Pmas fiber PAcK), mounted on the Calar Alto 3.5-m telescope. CALIFA DR3 provides widefield IFU data for 667 objects in total. The data for each galaxy are presented by two spectral datacubes that cover the spectral regions of 4300-7000 $\AA$ at a spectral resolution of $R \sim 850$ (setup V500) and of 3700-5000 $\AA$ at $R \sim 1650$ (setup V1200). Currently, there are 446 COMB datacubes that are a combination of V500 and V1200 datacubes and cover the spectral range of 3700-7000 $\mathrm{A}$. In this study we used COMB (a combination of V500+V1200) datacubes.

The galaxy inclination $i$ and position angle of the major axis PA were estimated from the analysis of the surface brightness image in the $r$ band of the SDSS. We applied the GALFIT code (Peng et al. 2002, 2010) to simultaenously fit the image of each galaxy with bulge+disk (Sersic+exponential profile) profiles. The position angle of the major axis and inclination of the exponential profile were adopted as the PA and $i$ for all galaxies, with the exception of NGC 1070. Because the fit of the exponential profile for NGC 1070 is unreliable, the PA and $i$ of the Sersic profile were adopted instead.

For further consideration, we selected galaxies with inclinations lower than $60^{\circ}$, which corresponds to a ratio of minor to major axis greater than $\sim 0.5$. The optical isophotal radius $R_{25}$ of a galaxy was determined from the analysis of the surface brightness profiles in the SDSS $g$ and $r$ bands converted into the Vega $B$ band. We used the stellar masses derived from UV-to-near-infrared (NIR) photometry, morphological type, bar, and merger classification from the parameter tables described in Walcher et al. (2014). We adopted the distances from the NED database with flow corrections for Virgo, the Great Attractor, and the Shapley Supercluster infall. The adopted distances, morphological types, PA, $i$, stellar masses, $R_{25}$, and $g-r$ colors are shown in Table 1.

We selected only galaxies with no merger features, that is, galaxies that are classified as isolated. Galaxies with insufficient numbers of spaxels with measured oxygen abundance were excluded from our sample. Thus, our final sample contains 66 galaxies with a radial oxygen abundance gradient derived by at least 10 spaxels that are distributed in a significantly wide radial range. Figure 1 shows the correlation between the stellar mass and the optical isophotal radius for our sample of galaxies.

\subsection{Emission line fluxes and abundance determination}

The spectrum of each spaxel from the CALIFA DR3 datacubes was processed in the same way as described in Zinchenko et al. (2016). Briefly, the stellar background in all spaxels was fit using the public version of the STARLIGHT code (Cid Fernandes et al. 2005; Mateus et al. 2006; Asari et al. 2007) adapted for execution in the NorduGrid ARC $^{1}$ environment of the Ukrainian National Grid. We used a set of 45 synthetic simple stellar population (SSP) spectra with metallicities $Z=0.004,0.02$, and

\footnotetext{
1 http://www .nordugrid.org/
} 
I. A. Zinchenko et al.: Oxygen abundance gradient in CALIFA galaxies

Table 1. Adopted and derived properties of our target galaxies.

\begin{tabular}{|c|c|c|c|c|c|c|c|c|c|c|c|c|}
\hline Name & $\begin{array}{r}\mathrm{D}^{a} \\
\mathrm{Mpc} \\
\end{array}$ & Type $^{b}$ & $\begin{array}{r}\text { PA } \\
\text { deg }\end{array}$ & $\begin{array}{r}i \\
\operatorname{deg} \\
\end{array}$ & $\begin{array}{r}\log M^{b} \\
M_{\odot} \\
\end{array}$ & $\begin{array}{l}R_{25} \\
\mathrm{kpc} \\
\end{array}$ & $\begin{array}{r}g-r \\
\mathrm{mag} \\
\end{array}$ & $\begin{array}{l}\log \Sigma_{0} \\
L_{\odot} / \mathrm{pc}^{2} \\
\end{array}$ & $A_{2}$ & $\begin{array}{c}\operatorname{grad}(\mathrm{O} / \mathrm{H}) \\
\operatorname{dex} / R_{25}\end{array}$ & $\begin{array}{c}\operatorname{grad}(\mathrm{O} / \mathrm{H})_{\mathrm{inn}} \\
\operatorname{dex} / R_{25}\end{array}$ & $\begin{array}{c}\operatorname{grad}(\mathrm{O} / \mathrm{H})_{\text {out }} \\
\operatorname{dex} / R_{25}\end{array}$ \\
\hline NGC 1 & 61.6 & SAbc & 102 & 41.4 & 10.629 & 11.65 & 0.517 & 2.339 & 0.080 & -0.082 & -0.185 & -0.065 \\
\hline NGC 23 & 61.7 & $\mathrm{SBb}$ & 155 & 59.3 & 10.980 & 18.13 & 0.514 & 2.420 & 0.216 & -0.127 & -0.113 & -0.116 \\
\hline NGC 171 & 52.8 & SBb & 98 & 25.8 & 10.447 & 15.21 & 0.454 & 2.586 & 0.294 & -0.230 & - & -0.230 \\
\hline NGC 180 & 70.6 & $\mathrm{SBb}$ & 159 & 50.2 & 10.658 & 23.21 & 0.395 & 2.157 & 0.188 & -0.172 & - & -0.189 \\
\hline NGC 214 & 61.0 & SABbc & 58 & 46.4 & 10.467 & 16.68 & 0.383 & 2.586 & 0.091 & -0.047 & - & - \\
\hline NGC 234 & 59.7 & $\mathrm{SABc}$ & 71 & 29.5 & 10.627 & 14.59 & 0.389 & 2.702 & 0.095 & -0.090 & -0.198 & -0.070 \\
\hline NGC 237 & 55.9 & $\mathrm{SBc}$ & 178 & 53.1 & 10.200 & 11.87 & 0.390 & 2.466 & 0.126 & -0.308 & -0.205 & -0.326 \\
\hline NGC 257 & 70.4 & SAc & 94 & 52.4 & 10.813 & 17.61 & 0.462 & 2.494 & 0.089 & -0.200 & 0.000 & -0.240 \\
\hline NGC 309 & 75.8 & SBcd & 112 & 25.8 & 10.743 & 25.80 & 0.297 & 2.450 & 0.154 & -0.331 & - & -0.355 \\
\hline NGC 477 & 89.5 & SABbc & 138 & 52.4 & 10.408 & 20.31 & 0.379 & 2.205 & 0.195 & -0.178 & -0.386 & -0.135 \\
\hline NGC 776 & 65.5 & $\mathrm{SBb}$ & 136 & 38.7 & 10.597 & 15.24 & 0.383 & 2.368 & 0.208 & -0.066 & -0.259 & -0.029 \\
\hline NGC 941 & 21.3 & SAcd & 175 & 28.4 & 9.153 & 6.51 & 0.175 & 2.155 & 0.133 & -0.328 & -0.181 & -0.309 \\
\hline NGC 976 & 57.4 & SAbc & 164 & 39.6 & 10.789 & 13.69 & 0.445 & 2.574 & 0.068 & -0.103 & -0.134 & -0.152 \\
\hline NGC 991 & 20.2 & SABcd & 126 & 18.2 & 9.577 & 7.29 & 0.320 & 2.254 & 0.096 & -0.336 & -0.607 & -0.307 \\
\hline NGC 1070 & 54.0 & $\mathrm{SAb}$ & $2^{c}$ & $33.9^{c}$ & 10.770 & 17.75 & 0.486 & 2.384 & 0.035 & -0.151 & -0.216 & 0.013 \\
\hline NGC 1094 & 85.8 & $\mathrm{SABb}$ & 95 & 56.6 & 10.642 & 18.22 & 0.419 & 2.120 & 0.172 & -0.168 & -0.041 & -0.188 \\
\hline NGC 1659 & 61.7 & SABbc & 49 & 51.0 & 10.425 & 15.08 & 0.387 & 2.560 & 0.132 & -0.237 & -0.290 & -0.190 \\
\hline NGC 1667 & 61.2 & $\mathrm{SBbc}$ & 172 & 45.6 & 10.714 & 15.13 & 0.372 & 2.960 & 0.131 & -0.069 & 0.065 & -0.086 \\
\hline NGC 2347 & 63.0 & SABbc & 3 & 54.6 & 10.543 & 15.76 & 0.412 & 2.409 & 0.112 & -0.283 & -0.258 & -0.318 \\
\hline NGC 2487 & 68.9 & $\mathrm{SBb}$ & 44 & 44.8 & 10.714 & 20.84 & 0.449 & 2.255 & 0.190 & 0.020 & - & 0.020 \\
\hline NGC 2530 & 73.0 & SABd & 152 & 34.9 & 9.988 & 14.86 & 0.232 & 2.160 & 0.222 & -0.273 & -0.601 & -0.217 \\
\hline NGC 2540 & 89.0 & $\mathrm{SBbc}$ & 126 & 47.9 & 10.495 & 15.53 & 0.433 & 2.384 & 0.154 & -0.170 & -0.437 & -0.157 \\
\hline NGC 2604 & 32.3 & SBd & 174 & 19.9 & 9.602 & 8.93 & 0.202 & 2.267 & 0.321 & -0.401 & -0.437 & -0.394 \\
\hline NGC 2730 & 56.7 & SBcd & 72 & 45.6 & 10.013 & 13.19 & 0.298 & 2.248 & 0.201 & -0.187 & -0.220 & -0.176 \\
\hline NGC 2805 & 28.7 & SAc & 175 & 30.7 & 9.730 & 16.28 & 0.134 & 1.569 & 0.146 & -0.229 & - & - \\
\hline NGC 2906 & 33.5 & SAbc & 82 & 56.6 & 10.412 & 8.19 & 0.352 & 2.741 & 0.107 & 0.004 & 0.028 & 0.000 \\
\hline NGC 2916 & 56.0 & SAbc & 16 & 49.5 & 10.514 & 16.94 & 0.406 & 2.628 & 0.160 & -0.132 & - & -0.132 \\
\hline NGC 3057 & 25.9 & SBdm & 2 & 56.6 & 9.024 & 7.53 & 0.134 & 1.741 & 0.148 & -0.229 & -0.493 & -0.164 \\
\hline NGC 3381 & 22.8 & SBd & 40 & 33.9 & 9.557 & 6.37 & 0.264 & 2.334 & 0.270 & -0.240 & -0.161 & -0.249 \\
\hline NGC 3614 & 38.4 & SABbc & 100 & 50.2 & 9.966 & 15.97 & 0.307 & 2.044 & 0.143 & -0.350 & -0.370 & -0.340 \\
\hline NGC 3687 & 41.1 & $\mathrm{SBb}$ & 150 & 23.1 & 10.156 & 10.64 & 0.437 & 2.485 & 0.135 & -0.251 & -0.033 & -0.279 \\
\hline NGC 3811 & 49.0 & $\mathrm{SBbc}$ & 14 & 48.7 & 10.321 & 12.97 & 0.426 & 2.530 & 0.121 & -0.144 & -0.341 & -0.141 \\
\hline NGC 4185 & 61.0 & SABbc & 167 & 47.9 & 10.600 & 18.81 & 0.496 & 2.279 & 0.094 & -0.297 & - & -0.297 \\
\hline NGC 4961 & 42.5 & SBcd & 111 & 50.9 & 9.574 & 8.90 & 0.236 & 2.173 & 0.142 & -0.339 & -0.442 & -0.299 \\
\hline NGC 5000 & 84.9 & SBbc & 69 & 48.7 & 10.485 & 17.04 & 0.420 & 2.308 & 0.273 & -0.092 & - & -0.032 \\
\hline NGC 5016 & 43.5 & SAbc & 58 & 46.4 & 10.202 & 11.64 & 0.433 & 2.503 & 0.065 & -0.143 & -0.105 & -0.245 \\
\hline NGC 5205 & 30.9 & $\mathrm{SBbc}$ & 157 & 53.8 & 9.943 & 7.55 & 0.451 & 2.385 & 0.160 & -0.032 & - & -0.032 \\
\hline NGC 5320 & 34.3 & SABbc & 14 & 58.0 & 10.156 & 11.17 & 0.340 & 2.316 & 0.094 & -0.248 & -0.304 & -0.245 \\
\hline NGC 5406 & 79.0 & $\mathrm{SBb}$ & 106 & 29.5 & 11.017 & 21.14 & 0.676 & 2.612 & 0.226 & -0.091 & - & -0.084 \\
\hline NGC 5480 & 32.9 & SAcd & 11 & 42.3 & 10.117 & 8.80 & 0.462 & 2.759 & 0.142 & -0.102 & -0.159 & -0.063 \\
\hline NGC 5520 & 33.2 & SAbc & 65 & 58.7 & 9.792 & 7.63 & 0.370 & 2.346 & 0.099 & -0.116 & -0.191 & -0.095 \\
\hline NGC 5633 & 39.6 & SAbc & 14 & 48.7 & 10.247 & 8.18 & 0.487 & 2.969 & 0.072 & -0.138 & -0.041 & -0.176 \\
\hline NGC 5720 & 113.1 & SBbc & 130 & 48.7 & 10.845 & 22.37 & 0.458 & 2.256 & 0.237 & -0.070 & - & -0.070 \\
\hline NGC 5732 & 59.3 & SAbc & 40 & 54.5 & 9.891 & 10.18 & 0.402 & 2.190 & 0.088 & -0.267 & -0.208 & -0.273 \\
\hline NGC 5888 & 126.7 & $\mathrm{SBb}$ & 158 & 56.0 & 11.153 & 23.22 & 0.626 & 2.408 & 0.065 & -0.039 & - & -0.039 \\
\hline NGC 5957 & 32.0 & $\mathrm{SBb}$ & 91 & 38.7 & 9.992 & 9.96 & 0.321 & 2.544 & 0.103 & -0.178 & - & -0.184 \\
\hline NGC 6004 & 60.8 & SBbc & 93 & 20.0 & 10.664 & 14.68 & 0.482 & 2.522 & 0.188 & -0.045 & -0.138 & -0.050 \\
\hline NGC 6063 & 46.7 & SAbc & 154 & 56.0 & 9.939 & 10.19 & 0.360 & 2.312 & 0.085 & -0.077 & - & -0.059 \\
\hline NGC 6154 & 88.7 & SBab & 137 & 48.7 & 10.771 & 18.84 & 0.552 & 2.179 & 0.173 & -0.032 & - & -0.032 \\
\hline NGC 6155 & 26.1 & $\mathrm{SAc}$ & 147 & 45.6 & 10.129 & 5.62 & 0.471 & 2.723 & 0.082 & -0.179 & -0.255 & -0.134 \\
\hline NGC 6301 & 120.3 & SAbc & 109 & 53.1 & 10.777 & 30.09 & 0.380 & 2.222 & 0.099 & -0.038 & - & -0.025 \\
\hline NGC 6497 & 84.8 & SBab & 116 & 57.3 & 10.934 & 16.53 & 0.613 & 2.324 & 0.275 & 0.004 & - & -0.000 \\
\hline NGC 6941 & 88.6 & $\mathrm{SBb}$ & 125 & 46.4 & 10.920 & 23.20 & 0.509 & 2.213 & 0.162 & -0.093 & - & -0.093 \\
\hline NGC 7321 & 97.9 & SBbc & 21 & 50.2 & 10.888 & 20.79 & 0.457 & 2.443 & 0.151 & -0.090 & - & -0.088 \\
\hline NGC 7364 & 67.1 & SAab & 64 & 51.7 & 10.807 & 14.44 & 0.521 & 2.292 & 0.073 & 0.005 & -0.040 & -0.064 \\
\hline NGC 7489 & 85.1 & SAbc & 164 & 56.6 & 10.341 & 22.28 & 0.097 & 1.968 & 0.115 & -0.414 & -0.278 & -0.400 \\
\hline NGC 7653 & 58.3 & $\mathrm{SAb}$ & 168 & 31.8 & 10.484 & 13.06 & 0.399 & 2.375 & 0.064 & -0.261 & -0.259 & -0.288 \\
\hline NGC 7716 & 35.6 & $\mathrm{SAb}$ & 31 & 39.6 & 10.289 & 10.04 & 0.494 & 2.457 & 0.098 & -0.120 & 0.108 & -0.109 \\
\hline NGC 7738 & 91.4 & $\mathrm{SBb}$ & 5 & 0.0 & 10.877 & 18.61 & 0.618 & 1.804 & 0.592 & -0.116 & - & -0.116 \\
\hline NGC 7819 & 67.2 & SAc & 88 & 53.1 & 10.085 & 14.27 & 0.312 & 1.884 & 0.216 & -0.295 & -0.184 & -0.326 \\
\hline IC 776 & 40.2 & SAdm & 92 & 53.8 & 9.277 & 8.65 & 0.202 & 1.590 & 0.209 & -0.174 & -0.525 & -0.068 \\
\hline IC 1256 & 72.1 & $\mathrm{SABb}$ & 93 & 51.7 & 10.252 & 14.47 & 0.381 & 2.288 & 0.089 & -0.294 & -0.355 & -0.304 \\
\hline IC 5309 & 57.4 & $\mathrm{SABc}$ & 26 & 59.3 & 10.222 & 9.68 & 0.487 & 2.043 & 0.098 & -0.188 & -0.859 & -0.160 \\
\hline UGC 8733 & 39.7 & SBdm & 6 & 58.0 & 9.314 & 10.51 & 0.196 & 1.701 & 0.206 & -0.267 & -0.218 & -0.203 \\
\hline UGC 12224 & 43.3 & SAc & 36 & 30.7 & 9.923 & 10.96 & 0.290 & 2.077 & 0.113 & -0.289 & - & -0.296 \\
\hline UGC 12816 & 71.9 & SAc & 142 & 54.5 & 9.379 & 13.59 & 0.167 & 1.822 & 0.111 & -0.201 & -0.582 & -0.071 \\
\hline
\end{tabular}

Notes. ${ }^{(a)}$ https: //ned.ipac. caltech.edu/ ${ }^{(b)}$ Walcher et al. (2014). ${ }^{(c)}$ Derived from Sersic profile.

0.05 , and 15 ages from 1 Myr up to 13 Gyr from the evolutionary synthesis models of Bruzual \& Charlot (2003) and the reddening law of Cardelli et al. (1989) with $R_{V}=3.1$. The resulting stellar radiation contribution was subtracted from the measured spectrum in order to determine the nebular emission spectrum. For each spectrum, we measured the fluxes of the [O II $] \lambda 3727+\lambda 3729, \mathrm{H} \beta,\left[\mathrm{O}_{\text {III }}\right] \lambda 4959,\left[\mathrm{O}_{\text {III }}\right] \lambda 5007,\left[\mathrm{~N}_{\text {II }}\right] \lambda 6548$,

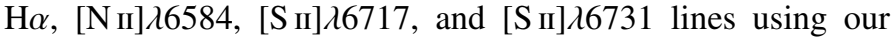
code ELF3D for emission line fitting. Each line was fit with a Gaussian profile. The measured line fluxes were corrected 


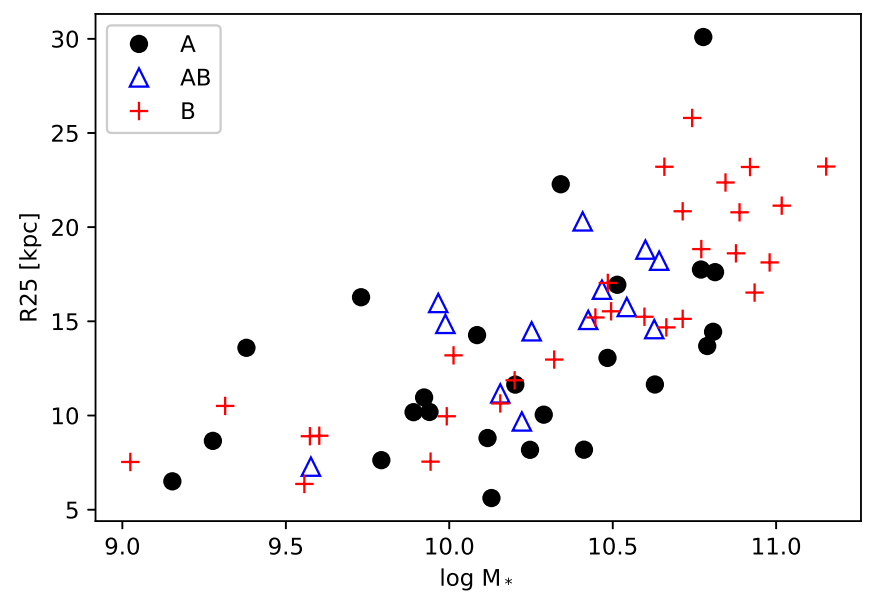

Fig. 1. Comparison of stellar mass and optical radius $R_{25}$ for our sample of galaxies. Unbarred (A), barred (B), and intermediate (AB) galaxies are shown with different colors and symbols.

for interstellar reddening using the theoretical $\mathrm{H} \alpha$ to $\mathrm{H} \beta$ ratio (i.e., the standard value of $\mathrm{H} \alpha / \mathrm{H} \beta=2.86$ ) and the analytical approximation of the Whitford interstellar reddening law from Izotov et al. (1994). When the measured value of $\mathrm{H} \alpha / \mathrm{H} \beta$ was lower than 2.86, the reddening was set to zero.

The $[\mathrm{O}$ III] $\lambda 5007$ and $\lambda 4959$ lines originate from transitions from the same energy level, therefore their flux ratio is determined only by the transition probability ratio, which is very close to 3 (Storey \& Zeippen 2000). The stronger line $\left[\mathrm{O}_{\mathrm{III}}\right] \lambda 5007$ is usually measured with higher precision than the weaker line [O $\mathrm{III}] \lambda 4959$. Therefore, the value of $R_{3}$ is estimated as $1.33[\mathrm{O}$ III] $\lambda 5007$, but not as a sum of the line fluxes. Similarly, the [N $\mathrm{II}] \lambda 6584$ and $\lambda 6548$ lines also originate from transitions from the same energy level, and the transition probability ratio for these lines is again close to 3 (Storey \& Zeippen 2000). The value of $N_{2}$ is therefore estimated as $1.33\left[\mathrm{~N}_{\mathrm{II}}\right] \lambda 6584$. Thus, the lines [O $\left.\mathrm{II}\right] \lambda \lambda$ 3727,3729, $\mathrm{H} \beta,\left[\mathrm{O}\right.$ III] $\lambda 5007, \mathrm{H} \alpha,\left[\mathrm{N}_{\mathrm{II}}\right] \lambda 6584$, [S II] 66717 , and [S II] 66731 are used for the dereddening and the abundance determinations. The precision of the line flux is specified by the ratio of the flux to the flux error (parameter $\epsilon$ ). We selected spectra where the parameter $\epsilon \geq 4$ for each of these lines. To select spaxels associated with star-forming regions, we applied BPT diagram $\log ([\mathrm{O}$ III $] \lambda 5007 / \mathrm{H} \beta)-\log ([\mathrm{N}$ II $] \lambda \lambda 6584 / \mathrm{H} \alpha)$ (Baldwin et al. 1981). To separate objects whose main ionization source are massive stars from those whose main ionization source are shocks of gas and/or active galactic nuclei (AGNs), we applied the criterion proposed by Kauffmann et al. (2003).

The CALIFA spectra, as well as the spectra of other surveys that use the IFU technique, can be contaminated by diffuse ionized gas (DIG; Haffner et al. 2009; Belfiore et al. 2015; Lacerda et al. 2018). This can lead to an increasing emission in low-ionization lines and therefore to biases in the chemical abundances derived by strong-line methods. However, Pilyugin et al. (2018) showed that the dividing line proposed by Kauffmann et al. (2003) can be applied to reject spectra with significant contamination by DIG.

To determine oxygen abundances, we used the new $R$ calibration developed by Pilyugin \& Grebel (2016). In comparison with previous calibrations, this calibration is applicable for the whole range of $\mathrm{H}$ II region metallicities and provides highprecision oxygen abundances with measurement errors smaller than 0.1 dex and a metallicity scale bounded to the direct $T_{\mathrm{e}}$

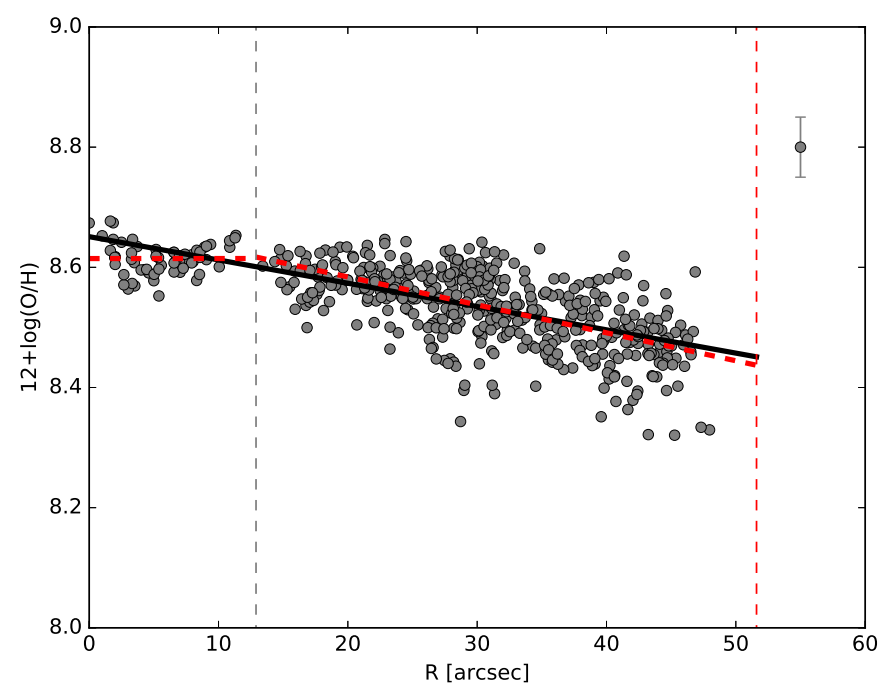

Fig. 2. Oxygen abundance gradient for NGC 257. Circles represent the oxygen abundance in the individual spaxels. Vertical dashed lines represent 0.25 and 1.0 fraction of optical radius $R_{25}$. The black solid line shows the best fit to all the data. Dashed red lines correspond to the best fit of the oxygen abundance gradient in the inner $\left(R<0.25 R_{25}\right)$ and outer $\left(0.25 R_{25}<R<R_{25}\right)$ parts of the galaxy. The typical uncertainty of the oxygen abundance determination for the single spaxel is presented in the top right corner.

method. Thus, the oxygen abundance was calculated for each spaxel using the R calibration.

\section{Oxygen abundance gradient}

We fit the radial oxygen abundance distribution in the disk using the following relation:

$$
12+\log (\mathrm{O} / \mathrm{H})=12+\log (\mathrm{O} / \mathrm{H})_{0}+\operatorname{grad} \times R,
$$

where $12+\log (\mathrm{O} / \mathrm{H})_{0}$ is the oxygen abundance at $R_{0}=0$, that is, the extrapolated value of the oxygen abundance in the galactic center, grad is the slope of the oxygen abundance gradient. It is known that the oxygen abundances in the inner and outer parts of some galaxies are systematically different from those expected from the overall abundance gradient (e.g., Belley \& Roy 1992; Bresolin et al. 2009, 2012; Goddard et al. 2011; Rosales-Ortega et al. 2011; Werk et al. 2011; Patterson et al. 2012; Sánchez et al. 2012, 2014; Sánchez-Menguiano et al. 2016, 2018; Zinchenko et al. 2016). However, within an optical radius, these differences are relatively small, smaller than $0.05 \mathrm{dex}$, and a break in the slope of the radial oxygen abundance distribution can be found at any radius (Pilyugin et al. 2017). Thus, the oxygen abundance gradient was estimated in three ranges of galactocentric distances: within $R_{25}$, in the inner $R<0.25 R_{25}$, and outer $0.25 R_{25}<R<R_{25}$ parts of the galaxy. It is presented in Table $1^{2}$.

Figure 2 shows the oxygen abundance gradient for NGC 257. Circles represent the oxygen abundance in the individual spaxels. The black solid line is the best fit to spaxels within the optical radius $R_{25}$ of the galaxy. Dashed red lines correspond to the best fit of the oxygen abundance gradient in the inner and outer parts of the galaxy. The radial gradient flattening is clearly seen in the inner part of the galaxy.

\footnotetext{
2 A detailed list of oxygen abundance gradients and errors for each galaxy is available at https://sites.google.com/view/ igorzinchenko/main/califa
} 

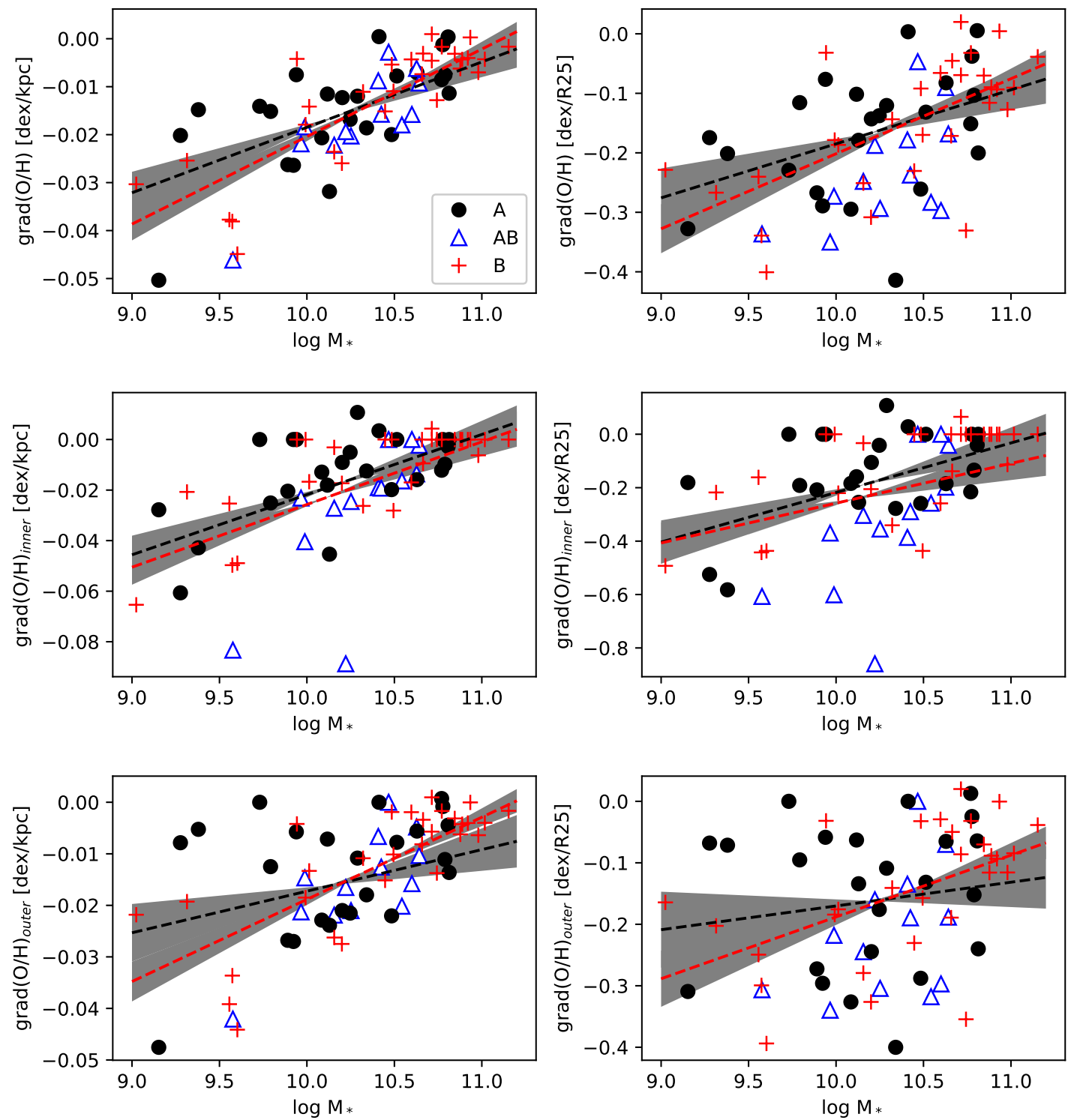

Fig. 3. Stellar mass - oxygen abundance gradient diagrams for the abundance gradient scaled to kpc (left columns) and $R_{25}$ (right columns). Top panels: oxygen abundance gradient for galactocentric distances $r<R_{25}$. Middle panels: oxygen abundance gradient for $r<0.25 R_{25}$. Bottom panels: oxygen abundance gradient for $0.25<r<R_{25}$. Unbarred (A), barred (B), and intermediate (AB) galaxies are shown with different colors and symbols. Black dashed lines are the best fit for the subsample of unbarred galaxies, and red lines show this for the barred galaxies. Shaded areas represent the $1 \sigma$ uncertainties of the fit.

Figure 3 shows the oxygen abundance gradient as a function of stellar mass. Black circles, red plus signs, and blue triangles represent unbarred $(\mathrm{A})$, barred $(\mathrm{B})$, and intermediate $(\mathrm{AB})$ galaxies, respectively. Black dashed lines are the best fit for the subsample of unbarred galaxies, and red lines show this for the barred galaxies. Shaded areas represent the $1 \sigma$ uncertainties of the fit. The left panels show the abundance gradient scaled to kiloparsecs, while the gradient in the right panels is scaled to $R_{25}$. In all cases there is no significant difference between barred and unbarred galaxies.

Galaxies with higher mass exhibit flatter oxygen abundance gradients when the gradient is measured per kpc (left panels of the Fig. 3), in agreement with the result of the numerical simulation of Tissera et al. (2016). This effect is significantly less prominent for the oxygen abundance gradients scaled to $R_{25}$ (right panels of the Fig. 3) and almost disappears for the gradient calculated in the outer region (bottom right panel). Our result confirms the conclusion of Ho et al. (2015), who attributed to the size effect the different dependence of the oxygen abundance gradient on the stellar mass for the oxygen abundance gradient normalized to $\mathrm{kpc}$ or $R_{25}$. They noted that galaxies with steeper oxygen abundance gradients usually have a smaller optical radius. Thus, our result suggests that at the present epoch, the variation in oxygen abundance between the central and the outer parts of galaxies is similar for galaxies in the whole mass range, while the radial variation in absolute scale (e.g., per kpc) is larger for less massive galaxies.

Different approaches to quantify the strength of the bar based on the axis ratio and bar length (Martin 1995; Martinet \& Friedli 1997; Chapelon et al. 1999; Aguerri 1999; Abraham \& Merrifield 2000; Marinova \& Jogee 2007), bar torque (Combes \& Sanders 1981; Buta et al. 2003; Laurikainen \& Salo 2002; Díaz-García et al. 2016), and Fourier analysis (Aguerri et al. 1998, 2000; Díaz-García et al. 2016; Garcia-Gómez et al. 2017), 

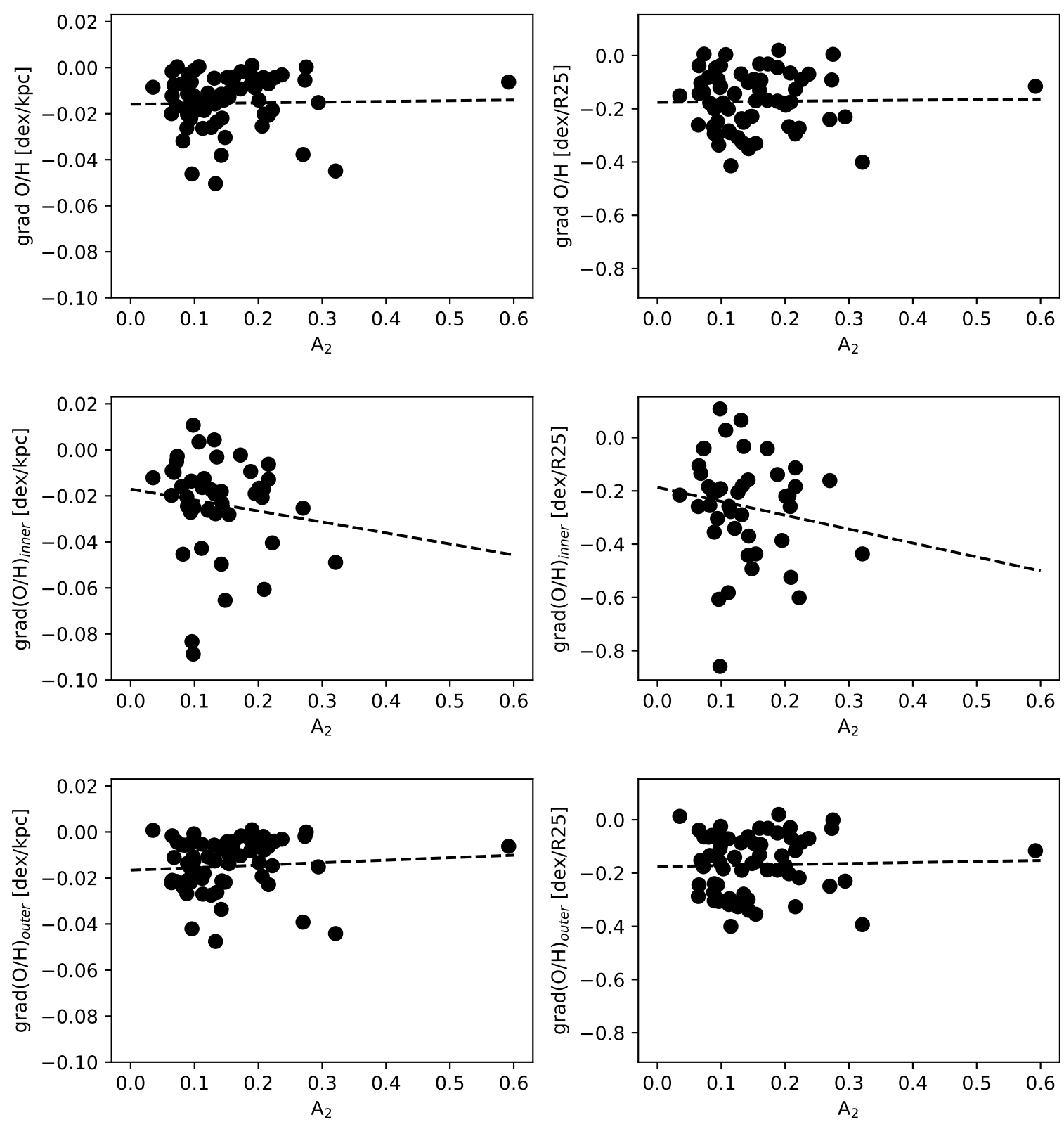

Fig. 4. Diagrams showing the $A_{2}$ Fourier coefficient - oxygen abundance gradient for the abundance gradient scaled to kpc (left columns) and $R_{25}$ (right columns). Top panels: oxygen abundance gradient for galactocentric distances $r<R_{25}$. Middle panels: oxygen abundance gradient for $r<0.25 R_{25}$. Bottom panels: oxygen abundance gradient for $0.25<r<R_{25}$.

for instance, have been suggested and used in the literature. Following Díaz-García et al. (2016) and Saha \& Naab (2013), to quantify the strength of the non-axisymmetric structures such as the bar and/or spiral patterns in the disk of a galaxy, we calculated the discrete Fourier transform of the surface brightness in the SDSS $r$ band in the disk of each galaxy. After correcting for inclination, we divided the galactic disk into concentric rings and defined the Fourier amplitude coefficient in each ring. The $m$ th complex Fourier coefficient is given by

$\tilde{A}_{m}\left(R_{k}\right)=\sum_{j=0}^{N-1} \mathrm{I}_{j} \mathrm{e}^{-2 \pi i m j / N}$,

where $I_{j}$ is the surface brightness within the $j$ th pixel in a given ring $R_{k} . A_{m}=\tilde{A}_{m} / \tilde{A}_{0}$ are the normalized Fourier amplitudes. $\tilde{A}_{0}$ refers to the axisymmetric surface brightness component (mean surface brightness at the given radius), and $\tilde{A}_{m}$ is an amplitude of the $m$ th mode of the azimuthal variation of surface brightness. For the further analysis, we adopted a maximum value of the $A_{2}$ Fourier amplitude for a galactocentric radius smaller than $0.5 R_{25}$, which corresponds to the maximum length of a bar (Erwin 2005). These derived maxima of the $A_{2}$ Fourier amplitudes for each galaxy are presented in Table 1 . We did not consider Fourier amplitudes in the center of galaxies $(R<2$ arcsec $)$ either because they can be affected by the uncertainties in the position of the galactic center.

Figure 4 shows the oxygen abundance gradient as a function of the maximum value of the $A_{2}$ Fourier amplitude. The dashed line is the linear fit of the data. We applied a two-sided $p$-value statistical test whose null hypothesis is that the slope of the grad versus $A_{2}$ relation is zero. The $p$-values range from 0.30 to 0.86 . We thus confirmed the null hypothesis of no correlation between oxygen abundance gradient and the $A_{2}$ Fourier amplitude of the surface brightness in the SDSS $r$ band. 
Table 2. Coefficients of parametric mass-metallicity relation.

\begin{tabular}{rccccccc}
\hline \hline$R$ & $c_{0}$ & $c_{1}$ & $c_{2}$ & $c_{R 25}$ & $c_{g-r}$ & $c_{\Sigma}$ & $c_{A 2}$ \\
\hline 0 & -2.7417 & 2.192 & -0.106 & 0.004 & 0.133 & 0.017 & -0.093 \\
$0.5 R_{25}$ & 0.7301 & 1.377 & -0.062 & -0.001 & 0.222 & 0.071 & 0.005 \\
$R_{25}$ & 6.2023 & 0.245 & -0.005 & -0.002 & 0.397 & 0.074 & -0.005 \\
\hline
\end{tabular}

\section{Generalized mass-metallicity relation}

The oxygen abundance in a galaxy can depend on the galaxy mass (Tremonti et al. 2004; Kewley \& Ellison 2008; Thuan et al. 2010), the surface brightness (Vila-Costas \& Edmunds 1992; Ryder 1995; Pilyugin et al. 2014b), the disk scale length (Pilyugin et al. 2014b; Zinchenko et al. 2015), the morphological type (Pilyugin et al. 2014b; Zinchenko et al. 2015), and the galactocentric radius (Peimbert 1979; Shields \& Searle 1978; Sánchez et al. 2014; Zinchenko et al. 2016; Belfiore et al. 2017, among many others). Moreover, the dependence on the given parameter can be seen only at some range of galactocentric distances (Pilyugin et al. 2014b). We therefore considered the generalized mass-metallicity relation as a multiparameter function to distinguish the impact of size, color, disk surface brightness, and strength of bar/spirals on the oxygen abundance in the central, intermediate, and outer parts of the galaxy.

For a given galactocentric radius we applied the following parametric relation:

$$
\begin{aligned}
12+\log (\mathrm{O} / \mathrm{H}) & =c_{0}+c_{1} \log M_{*}+c_{2}\left(\log M_{*}\right)^{2} \\
& +c_{R 25} R_{25}+c_{g-r}(g-r) \\
& +c_{\Sigma} \log \Sigma_{0}+c_{A 2} A_{2}
\end{aligned}
$$

where $M_{*}$ is the stellar mass of the galaxy expressed in the mass of the Sun, $R_{25}$ is the isophotal radius of a galaxy in kpc, $g-r$ is the difference between the total magnitude of the galaxy in the SDSS $g$ and $r$ bands, $\Sigma_{0}$ is the central surface brightness of the disk in the SDSS $r$ band in units of $L_{\odot} / \mathrm{pc}^{2}$, and $A_{2}$ is the maximum value of the $A_{2}$ Fourier coefficient. This relation was constructed for the three galactocentric radii of $0,0.5 R_{25}$, and $R_{25}$. The oxygen abundance at a given radius was calculated using the fit of the abundance gradient. Abundances at the center of each galaxy were obtained from the inner region gradient fit $\left(R<R_{25}\right)$, while oxygen abundances at $R=0.5 R_{25}$ and $R=R_{25}$ were estimated using the outer gradient fit $\left(0.25 R_{25}<R<R_{25}\right)$. The values of each coefficient for the three considered radii are listed in Table 2.

The disk surface brightness was obtained in the following way. We corrected the surface brightness of each pixel for Galactic foreground extinction using the given in the NASA Extragalactic Database (NED) ${ }^{3}$. We applied the $A_{r}$ values from the recalibration by Schlafly \& Finkbeiner (2011) of the Schlegel et al. (1998) infrared-based dust map. Then, we fit the radial surface brightness profile by an exponential profile

$\Sigma(R)=\Sigma_{0} \exp (-R / h)$,

where $\Sigma_{0}$ is the central disk surface brightness and $h$ is the radial scale length. As the radial surface brightness profiles of many galaxies are affected by the bulge in the central part, we fit the surface brightness for the galactocentric distances $0.3 R_{25}<R<$ $R_{25}$. The surface brightness was converted into solar units for the further analysis. The magnitude of the Sun in the SDSS $r$ band $M_{\odot, r}=4.64$ was taken from Blanton \& Roweis (2007).

\footnotetext{
3 https://ned.ipac.caltech.edu/
}

We estimated the probability distribution function (PDF) and the $95 \%$ confidence intervals for the $c_{R 25}, c_{g-r}, c_{\Sigma}$, and $c_{A 2}$ coefficients of the generalized mass-metallicity relation using a bootstrap method with 100000 iterations (Fig. 5). We considered the generalized mass-metallicity relation for the local oxygen abundance in the center, at the half of the optical radius, and at the optical radius of a galaxy. For the central part of a galaxy, the zero value falls within the $95 \%$ confidence interval of the PDF for all parameters. This shows that the oxygen abundance in the central part of a galaxy depends neither on the optical radius $R_{25}$ nor on the color $g-r, \Sigma_{0}$, and $A_{2}$ of the galaxy. However, outside the central part, the oxygen abundance increases with $g-r$ and $\Sigma_{0}$ at a confidence level of almost $95 \%$. The right side columns of Fig. 5 show that there is no dependence of the generalized mass-metallicity relation on the $A_{2}$ parameter because the zero value falls within the $95 \%$ confidence interval of the PDF at all radii.

Figure 6 shows the probability distribution function for the $c_{1}$ (left column) and $c_{2}$ (middle column) coefficients of the generalized mass-metallicity relation in the center of galaxies (top panels), at $R=0.5 R_{25}$ (middle panels), and $R=R_{25}$ (bottom panels). The solid vertical line represents the zero value for each coefficient. The coefficient $c_{2}$ represents the nonlinearity of the generalized mass-metallicity relation, which is significant in the inner region of galaxies. The right column panels, which are 2D PDFs in $c_{1}$ and $c_{2}$ coordinate axes, represent the strong correlation between the $c_{1}$ and $c_{2}$ coefficients at all galactocentric radii. Because of this and the short mass range, the width of the PDF of $c_{1}$ and $c_{2}$ coefficients is quite large. For the outer part of the galaxy, the PDF centers for the $c_{1}$ and $c_{2}$ coefficients are close to zero. This might be thought to indicate that in the outer parts of the galaxies the oxygen abundance does not depend on the galaxy mass. To determine whether this is true, we examined the right bottom panel, which represents the correlation between $c_{1}$ and $c_{2}$ for the outer region of a disk. This panel contains the zoomed central part of the distribution. It is very unlikely that the $c_{1}$ and $c_{2}$ coefficients are both equal to zero simultaneously. This means that the oxygen abundance depends on the galaxy mass at the outer part of a galaxy as well.

We here limit our sample to face-on galaxies of large angular size in order to resolve the bar and spiral structure. In a future work we will investigate the nonlinearity of the generalized mass-metallicity relation in detail using a larger galaxy sample.

\section{Discussion and summary}

We investigated the possible dependence of the oxygen abundance gradient in disk galaxies on the presence of a bar/spiral and other parameters of the galaxy, such as mass, size, color index, and surface brightness. We also studied the generalized mass-metallicity relation for the oxygen abundance in the center, intermediate, and outer parts of the galaxy as a multivariable relation including size, color index, surface brightness, and 

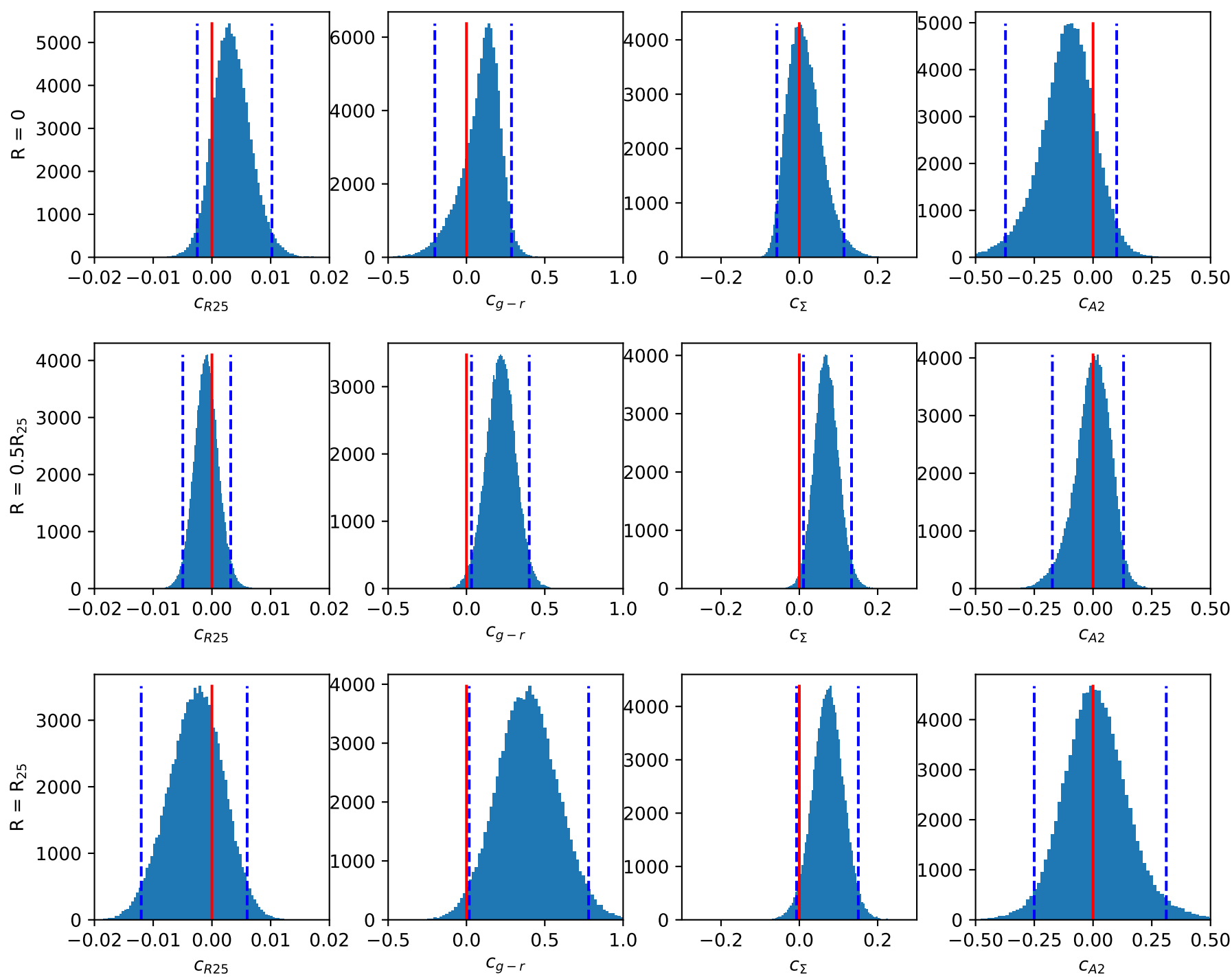

Fig. 5. Probability distribution function for the $c_{R 25}, c_{g-r}, c_{\Sigma}$, and $c_{A 2}$ coefficients of the generalized mass-metallicity relation in the center of galaxies (top panels), at $R=0.5 R_{25}$ (middle panels), and $R=R_{25}$ (bottom panels). The solid vertical line represents the zero value for each coefficient, and the dashed lines show the two-sided $95 \%$ confidence interval.

the bar/spiral pattern strength. Our sample contains 66 galaxies from the CALIFA DR3 survey. We adopted the Fourier amplitude $A_{2}$ of the surface brightness as the quantitative characteristic of the strength of non-axisymmetric structures in a galactic disk, in addition to a commonly used morphologic division into A, AB, and B types based on the Hubble classification.

We considered the generalized mass-metallicity relation in order to take into account a possible dependence of the local oxygen abundance and the strength of the non-axisymmetric structures in a disk. Our relation also takes into account the influence of the isophotal radius $R_{25}$, the color index $g-r$ in the SDSS bands, the central surface brightness of the disk $\Sigma$ in the SDSS $r$ band, and the bar/spiral strength, quantified as the maximum amplitude of the $\mathrm{A}_{2}$ Fourier coefficient. This approach allowed us to distinguish changes in local oxygen abundance that are caused by each parameter even when the oxygen abundance depends on many parameters simultaneously. This relation was constructed for the three galactocentric radii of $0,0.5 R_{25}$, and $R_{25}$.

We find larger negative oxygen abundance gradients scaled to $\mathrm{kpc}$ for the galaxies with lower masses. However, this effect is significantly less prominent for the oxygen abundance gradi- ents scaled to $R_{25}$, as has been shown by previous observational (Ho et al. 2015) and theoretical (Prantzos \& Boissier 2000) studies. The dependence of oxygen abundance gradient on the stellar mass almost disappears when the gradient is calculated for the outer part of the galaxy and scaled to $R_{25}$.

We find neither a significant difference between the oxygen abundance gradient in unbarred and barred galaxies (Hubble types A an B) nor a dependence of the mass-local metallicity relation on the $A_{2}$ parameter at any galactocentric radius. A standard deviation of the difference between oxygen abundances, observed and calculated with our generalized mass-metallicity relation, ranges from $0.04 \mathrm{dex}$ at the center to $0.07 \mathrm{dex}$ at the optical radius. Thus, our data suggest that there is no significant impact of the non-axisymmetric structures such as a bar and/or spiral patterns on the oxygen abundance and radial metallicity gradient of the spiral galaxies. The effect of the oxygen abundance distribution is smaller than $\sim 0.05 \mathrm{dex}$ at any distance from the center of a galaxy. This conclusion agrees with the results on the stellar (Sánchez-Blázquez et al. 2014; Cheung et al. 2015) and gas-phase (Sánchez-Menguiano et al. 2016) metallicity gradients as well as with a few recent studies of 

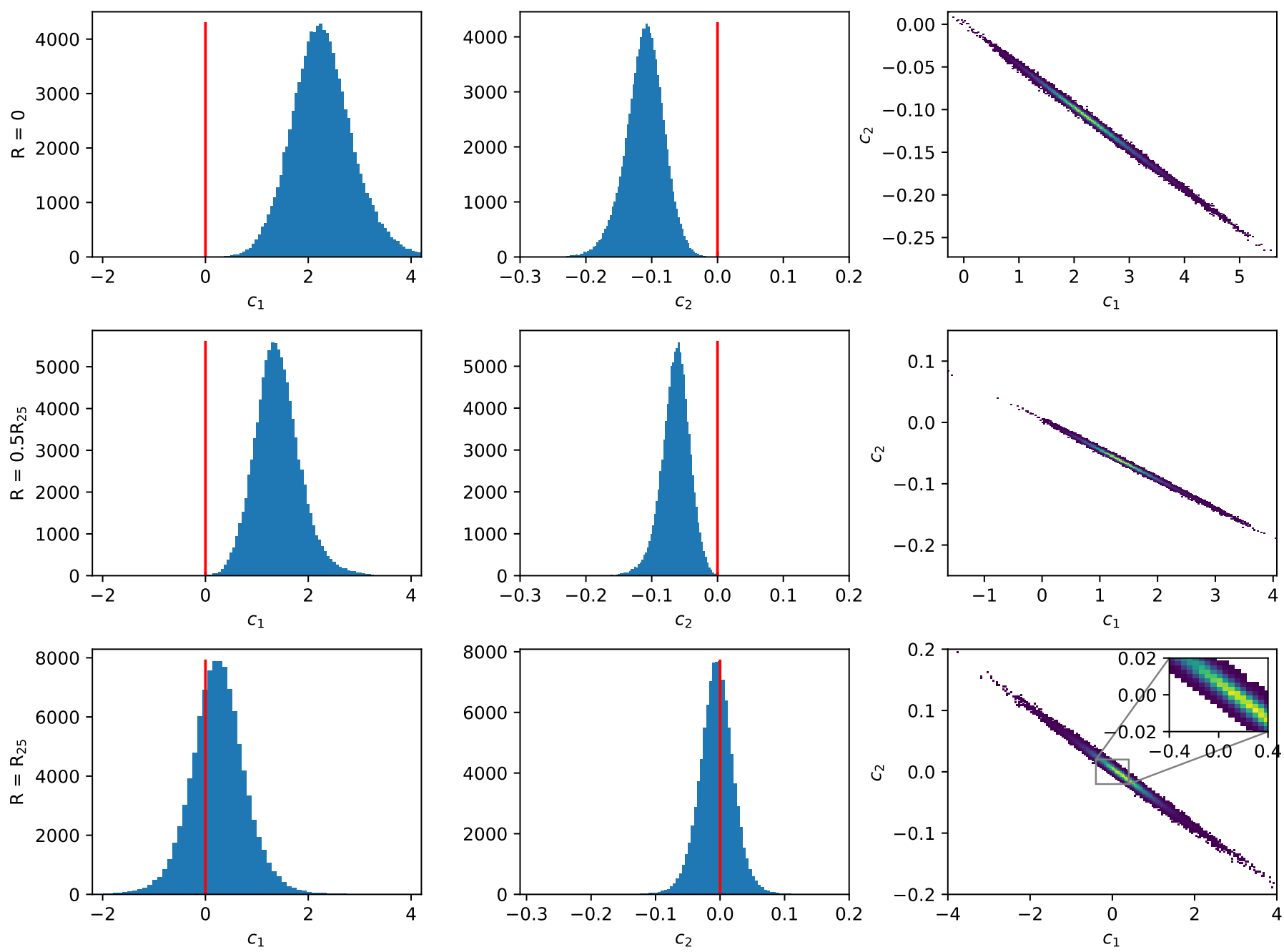

Fig. 6. Probability distribution function for the $c_{1}$ (left column) and $c_{2}$ (middle column) coefficients of the generalized mass-metallicity relation in the center of galaxies (top panels), at $R=0.5 R_{25}$ (middle panels), and $R=R_{25}$ (bottom panels). The solid vertical line represents the zero value for each coefficient. The right column represents the correlation between the $c_{1}$ and $c_{2}$ coefficients.

the azimuthal variation in oxygen abundance (in the spiral arms and interarm medium) for the CALIFA galaxies. Sakhibov et al. (2018) showed that the enhancement of the oxygen abundance in the spiral arms as compared to the interarm regions is lower that 0.05 dex. Moreover, no significant changes in radial oxygen abundance gradient between the spiral arms and the interarm medium have been found. In addition, Sánchez-Menguiano et al. (2017) noted some differences in the azimuthal distribution of the oxygen abundance between the arm and interarm starforming regions in barred and flocculent spirals. They suggested that this influence is not strong enough to affect the overall abundance distribution, which is confirmed by our results.

The absence of a correlation between the gas-phase oxygen abundance and the non-axisymmetric structures in galaxy disks suggests that at least for the oxygen, the processes of chemical enrichment of the interstellar medium are more efficient in producing the radial abundance gradient than the gas mixing.

We find that the local oxygen abundance in the outer part of the disk increased with the central surface brightness of the disk, while there is no such correlation for the central part of the galaxies. This result is consistent with the conclusion of Wu et al. (2015). They considered the averaged oxygen abundance in nearby galaxies with respect to its surface brightness and found that galaxies with lower surface brightness tend to have lower metallicity. The correlation between local oxygen abundance and surface brightness, in addition to the flattening of the radial metallicity gradient in the massive galaxies, can be considered as confirmation of the inside-out galaxy formation scenario (Kepner 1999; Pilkington et al. 2012). If the chemical evolution in the central part of the galaxy is almost completed, the dependence of the metallicity on the galaxy parameters should indeed be suppressed.

Our findings are briefly summarized below.

1. We showed that galaxies with higher stellar mass exhibit flatter oxygen abundance gradients. This correlation becomes more prominent for gradients expressed in units of dex/kpc and obtained for the central part of the galaxies $(R<$ $0.25 R_{25}$ ) and for the galactocentric distances in the range from 0 to $R_{25}$.

2. There is no significant difference between the oxygen abundance gradient in unbarred and barred galaxies (Hubble types A and B). In addition, we did not find a dependence of the generalized mass-metallicity relation on the $A_{2}$ parameter. Our data suggest that there is no significant impact of the non-axisymmetric structures such as a bar and/or spiral patterns on the local oxygen abundance in the disk of a galaxy or this impact is weaker than $\sim 0.05$ dex. However, this correlation is significantly reduced when the abundance gradients 
are scaled to $R_{25}$, and it almost disappears when the gradients are calculated for the outer part of the galaxy.

3. The local oxygen abundance does not depend on the optical radius $R_{25}$ at any radius.

4. The local oxygen abundance increases with color index $g-r$ of the galaxy outside the inner part.

5. The local oxygen abundance depends on the central surface brightness of the disk $\Sigma_{0}$ only in the outer region of the galaxy. The correlation between local oxygen abundance and surface brightness in the outer region of galaxies, in addition to the flattening of the radial metallicity gradient in the massive galaxies, can be considered as confirmation of the inside-out galaxy formation scenario (Kepner 1999; Pilkington et al. 2012).

Acknowledgements. We are grateful to the referee for their constructive comments. I.A.Z. thanks the German Academic Exchange Service (DAAD) for financial support. I.A.Z. and L.S.P. acknowledge support within the framework of Sonderforschungsbereich (SFB 881) on "The Milky Way System" (especially subproject A5), which is funded by the German Research Foundation (DFG). L.S.P. and I.A.Z. thank the Astronomisches Rechen-Institut at the Universität Heidelberg, where this investigation was carried out, for the hospitality. I.A.Z. acknowledges the support by the Ukrainian National Grid program (project 400Kt) of the NAS of Ukraine. This study uses data provided by the Calar Alto Legacy Integral Field Area (CALIFA) survey (http://califa. caha.es/). Based on observations collected at the Centro Astronomico Hispano Aleman (CAHA) at Calar Alto, operated jointly by the Max-Planck-Institut fur Astronomie and the Instituto de Astrofisica de Andalucia (CSIC).

\section{References}

Abraham, R. G., \& Merrifield, M. R. 2000, AJ, 120, 2835

Aguerri, J. A. L. 1999, A\&A, 351, 43

Aguerri, J. A. L., Beckman, J. E., \& Prieto, M. 1998, AJ, 116, 2136

Aguerri, J. A. L., Muñoz-Tuñón, C., Varela, A. M., \& Prieto, M. 2000, A\&A, 361,841

Asari, N. V., Cid Fernandes, R., Stasińska, G., et al. 2007, MNRAS, 381, 263

Athanassoula, E. 1992, MNRAS, 259, 345

Baldwin, J. A., Phillips, M. M., \& Terlevich, R. 1981, PASP, 93, 5

Belfiore, F., Maiolino, R., Bundy, K., et al. 2015, MNRAS, 449, 867

Belfiore, F., Maiolino, R., Tremonti, C., et al. 2017, MNRAS, 469, 151

Belley, J., \& Roy, J.-R. 1992, ApJS, 78, 61

Blanton, M. R., \& Roweis, S. 2007, AJ, 133, 734

Blanton, M. R., Bershady, M. A., Abolfathi, B., et al. 2017, AJ, 154, 28

Bresolin, F., Ryan-Weber, E., Kennicutt, R. C., \& Goddard, Q. 2009, ApJ, 695, 580

Bresolin, F., Kennicutt, R. C., \& Ryan-Weber, E. 2012, ApJ, 750, 122

Bruzual, G., \& Charlot, S. 2003, MNRAS, 344, 1000

Bundy, K., Bershady, M. A., Law, D. R., et al. 2015, ApJ, 798, 7

Buta, R., Block, D. L., \& Knapen, J. H. 2003, AJ, 126, 1148

Cardelli, J. A., Clayton, G. C., \& Mathis, J. S. 1989, ApJ, 345, 245

Chapelon, S., Contini, T., \& Davoust, E. 1999, A\&A, 345, 81

Cheung, E., Conroy, C., Athanassoula, E., et al. 2015, ApJ, 807, 36

Cid Fernandes, R., Mateus, A., Sodré, L., Stasińska, G., \& Gomes, J. M. 2005, MNRAS, 358, 363

Combes, F., \& Sanders, R. H. 1981, A\&A, 96, 164

Díaz-García, S., Salo, H., Laurikainen, E., \& Herrera-Endoqui, M. 2016, A\&A, 587, A160

Di Matteo, P., Haywood, M., Combes, F., Semelin, B., \& Snaith, O. N. 2013, A\&A, 553, A102

Erwin, P. 2005, MNRAS, 364, 283

Garcia-Gómez, C., Athanassoula, E., Barberà, C., \& Bosma, A. 2017, A\&A, 601, A132

Garnett, D. R. 1998, Rev. Mex. Astron. Astrofis. Conf. Ser., 7, 58

Goddard, Q. E., Bresolin, F., Kennicutt, R. C., Ryan-Weber, E. V., \& Rosales-Ortega, F. F. 2011, MNRAS, 412, 1246

Grand, R. J. J., \& Kawata, D. 2016, Astron. Nachr., 337, 957

Haffner, L. M., Dettmar, R.-J., Beckman, J. E., et al. 2009, Rev. Mod. Phys., 81, 969

Ho, I.-T., Kudritzki, R.-P., Kewley, L. J., et al. 2015, MNRAS, 448, 2030
Izotov, Y. I., Thuan, T. X., \& Lipovetsky, V. A. 1994, ApJ, 435, 647

Kauffmann, G., Heckman, T. M., Tremonti, C., et al. 2003, MNRAS, 346, 1055

Kepner, J. V. 1999, ApJ, 520, 59

Kewley, L. J., \& Ellison, S. L. 2008, ApJ, 681, 1183

Lacerda, E. A. D., Cid Fernandes, R., Couto, G. S., et al. 2018, MNRAS, 474, 3727

Laurikainen, E., \& Salo, H. 2002, MNRAS, 337, 1118

Lian, J., Thomas, D., Maraston, C., et al. 2018, MNRAS, 476, 3883

Marinova, I., \& Jogee, S. 2007, ApJ, 659, 1176

Martin, P. 1995, AJ, 109, 2428

Martin, P., \& Roy, J.-R. 1994, ApJ, 424, 599

Martinet, L., \& Friedli, D. 1997, A\&A, 323, 363

Mateus, A., Sodré, L., Cid Fernandes, R., et al. 2006, MNRAS, 370, 721

Mayor, M. 1976, in Abundance Effects in Classification, eds. B. Hauck,

P. C. Keenan, \& W. W. Morgan , IAU Symp., 72, 207

McCall, M. L., Rybski, P. M., \& Shields, G. A. 1985, ApJS, 57, 1

Minchev, I., \& Famaey, B. 2010, ApJ, 722, 112

Minchev, I., Chiappini, C., \& Martig, M. 2013, A\&A, 558, A9

Minchev, I., Chiappini, C., \& Martig, M. 2014, A\&A, 572, A92

Patterson, M. T., Walterbos, R. A. M., Kennicutt, R. C., Chiappini, C., \& Thilker, D. A. 2012, MNRAS, 422, 401

Peimbert, M. 1979, in The Large-Scale Characteristics of the Galaxy, ed W. B. Burton, IAU Symp., 84, 307

Peng, C. Y., Ho, L. C., Impey, C. D., \& Rix, H.-W. 2002, AJ, 124, 266

Peng, C. Y., Ho, L. C., Impey, C. D., \& Rix, H.-W. 2010, AJ, 139, 2097

Pilkington, K., Few, C. G., Gibson, B. K., et al. 2012, A\&A, 540, A56

Pilyugin, L. S., \& Grebel, E. K. 2016, MNRAS, 457, 3678

Pilyugin, L. S., Grebel, E. K., \& Kniazev, A. Y. 2014a, AJ, 147, 131

Pilyugin, L. S., Grebel, E. K., Zinchenko, I. A., \& Kniazev, A. Y. 2014b, AJ, 148,134

Pilyugin, L. S., Grebel, E. K., Zinchenko, I. A., Nefedyev, Y. A., \& Vílchez, J. M. 2017, A\&A, 608, A127

Pilyugin, L. S., Grebel, E. K., Zinchenko, I. A., et al. 2018, A\&A, 613, A1

Prantzos, N., \& Boissier, S. 2000, MNRAS, 313, 338

Rich, J. A., Torrey, P., Kewley, L. J., Dopita, M. A., \& Rupke, D. S. N. 2012, ApJ, 753, 5

Rosales-Ortega, F. F., Díaz, A. I., Kennicutt, R. C., \& Sánchez, S. F. 2011, MNRAS, 415, 2439

Roy, J. R. 1996, in IAU Colloq. 157: Barred Galaxies, eds. R. Buta, D. A. Crocker, \& B. G. Elmegreen, ASP Conf. Ser., 91, 63

Ruiz-Lara, T., Pérez, I., Florido, E., et al. 2017, A\&A, 604, A4

Ryder, S. D. 1995, ApJ, 444, 610

Saha, K., \& Naab, T. 2013, MNRAS, 434, 1287

Sakhibov, F., Zinchenko, I. A., Pilyugin, L. S., et al. 2018, MNRAS, 474, 1657

Sánchez, S. F., Kennicutt, R. C., Gil de Paz, A., et al. 2012, A\&A, 538, A8

Sánchez, S. F., Rosales-Ortega, F. F., Iglesias-Páramo, J., et al. 2014, A\&A, 563, A49

Sánchez, S. F., García-Benito, R., Zibetti, S., et al. 2016, A\&A, 594, A36

Sánchez-Blázquez, P., Rosales-Ortega, F. F., Méndez-Abreu, J., et al. 2014, A\&A, 570, A6

Sánchez-Menguiano, L., Sánchez, S. F., Pérez, I., et al. 2016, A\&A, 587, A70

Sánchez-Menguiano, L., Sánchez, S. F., Pérez, I., et al. 2017, A\&A, 603, A113

Sánchez-Menguiano, L., Sánchez, S. F., Pérez, I., et al. 2018, A\&A, 609, A119

Schlafly, E. F., \& Finkbeiner, D. P. 2011, ApJ, 737, 103

Schlegel, D. J., Finkbeiner, D. P., \& Davis, M. 1998, ApJ, 500, 525

Schönrich, R., \& Binney, J. 2009, MNRAS, 396, 203

Searle, L. 1971, ApJ, 168, 327

Sellwood, J. A., \& Binney, J. J. 2002, MNRAS, 336, 785

Shields, G. A., \& Searle, L. 1978, ApJ, 222, 821

Storey, P. J., \& Zeippen, C. J. 2000, MNRAS, 312, 813

Thuan, T. X., Pilyugin, L. S., \& Zinchenko, I. A. 2010, ApJ, 712, 1029

Tissera, P. B., Pedrosa, S. E., Sillero, E., \& Vilchez, J. M. 2016, MNRAS, 456, 2982

Tremonti, C. A., Heckman, T. M., Kauffmann, G., et al. 2004, ApJ, 613, 898

Vila-Costas, M. B., \& Edmunds, M. G. 1992, MNRAS, 259, 121

Walcher, C. J., Wisotzki, L., Bekeraité, S., et al. 2014, A\&A, 569, A1

Werk, J. K., Putman, M. E., Meurer, G. R., \& Santiago-Figueroa, N. 2011, ApJ, 735, 71

Wu, P.-F., Kudritzki, R.-P., Tully, R. B., \& Neill, J. D. 2015, ApJ, 810, 151

Zaritsky, D., Kennicutt, Jr., R. C., \& Huchra, J. P. 1994, ApJ, 420, 87

Zinchenko, I. A., Kniazev, A. Y., Grebel, E. K., \& Pilyugin, L. S. 2015, A\&A, 582, A35

Zinchenko, I. A., Pilyugin, L. S., Grebel, E. K., Sánchez, S. F., \& Vílchez, J. M. 2016, MNRAS, 462, 2715 